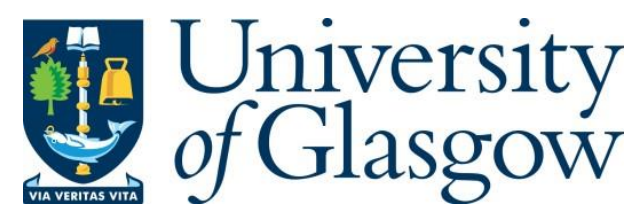

Melino, G. et al. (2020) Capsule endoscopy compatible fluorescence imager demonstrated using bowel cancer tumours. IEEE Sensors Journal, (doi:

10.1109/JSEN.2020.2990293).

There may be differences between this version and the published version. You are advised to consult the publisher's version if you wish to cite from it.

http://eprints.gla.ac.uk/214885/

Deposited on: 11 May 2020

Enlighten - Research publications by members of the University of Glasgow http://eprints.gla.ac.uk 


\title{
Capsule Endoscopy Compatible Fluorescence Imager Demonstrated Using Bowel Cancer Tumours
}

\author{
Gianluca Melino ${ }^{1}$, Claudio Accarino ${ }^{1}$, Mathis Riehle ${ }^{2}$, Mark Potter ${ }^{3}$, Paul Fineron ${ }^{4}$, Valerio F. \\ Annese ${ }^{1}$, James P. Grant ${ }^{1}$, Mohammed Al-Rawhani ${ }^{1}$, James Beeley ${ }^{1}$, Ivonne Escorcia Carranza ${ }^{1}$ and \\ David R.S. Cumming ${ }^{1}$, Fellow, IEEE
}

\begin{abstract}
We demonstrate a proof of concept highly miniaturised fluorescence imager and its application to detecting cancer in resected human colon cancer tissues. Fluorescence imaging modalities have already been successfully implemented in traditional endoscopy. However, the procedure still causes discomfort and requires sedation. Wireless fluorescence capsule endoscopy has the potential to improve diagnostic accuracy with less inconvenience for patients. In this paper we present a $5 \mathrm{~mm} x$ $6 \mathrm{~mm} \times 5 \mathrm{~mm}$ optical block that is small enough to integrate into a capsule endoscope. The block integrates ultrathin filters for optical isolation and was successfully integrated with a sensitive CMOS SPAD array to detect green fluorescence from Flavin Adenine Dinucleotide (FAD), which is an endogenous fluorophore responsible for autofluorescence in human tissues, and fluorescence from the cancer selective molecular probe ProteoGREEN ${ }^{\mathrm{TM}}$-gGlu used to label colorectal cancer cells. In vitro studies were validated using a commercial Modulus ${ }^{\mathrm{TM}}$ Microplate reader. The potential use of the device in capsule endoscopy was further validated by imaging healthy and malignant resected human tissues from the colon to detect changes in autofluorescence signal that are crucial for cancer diagnosis.
\end{abstract}

Index Terms - early diagnosis, miniaturisation, capsule endoscopy, fluorescence imaging, bowel cancer, autofluorescence, fluorescence labelling, CMOS, SPAD, ultra-thin optical filters

\section{INTRODUCTION}

$\mathrm{I}_{\mathrm{a} n \mathrm{n}}$ N 2001, the U.S. Food and Drug Administration (FDA) approved the use of capsule endoscopy (CE) as an auxiliary method for the investigation of the small bowel; otherwise inaccessible by traditional endoscopic procedures[1]. Clinicians have also shown the use of CE in the large bowel for determining the location of several gastrointestinal (GI) diseases such as Obscure Gastrointestinal Bleeding (OGIB), malignant or benign neoplastic lesions (polyps) and celiac disease [2]. The optical system of CE usually consists of white light emitting diodes (LEDs), short focal length lenses and a CMOS image sensor [1].

\footnotetext{
${ }^{1}$ School of Engineering, University of Glasgow, Glasgow, G12 8LT, UK. ${ }^{2}$ Centre for Cell Engineering, School of Chemistry, University of Glasgow, Glasgow, G12 8QQ, United Kingdom.

${ }^{3}$ Colorectal Surgical Unit, Alexander Donald Building, Western General Hospital, Crew Road, University of Edinburgh, EH4 2XU, United Kingdom.
}

In the early stages of cancer, many lesions cause no substantial morphological changes in the GI walls and are undetectable by white light imaging (WLI)[3]. As a result, diagnosis might become inaccurate. A better detection and classification of suspicious areas in the GI mucosa can be achieved by using new imaging modalities such as autofluorescence imaging (AFI)[4]. This technique has been successfully implemented in traditional endoscopes exploiting a fibre optic bundle connected to external units to obtain a fluorescence image [5].

In AF endoscopy, fluorescence light is detected by exploiting the natural fluorescence of human tissues with blue light illumination[6]. Healthy areas will emit green autofluorescence while diseased areas will appear as darker regions [4], [7], [8].

A different methodology involves targeting lesions with cancer-selective molecular fluorescence probes[9]-[11]. Specific probes are not intrinsically fluorescent but undergo reactions with metabolites, overexpressed in carcinogenic environment, that change their molecular structures producing highly fluorescent products [9], [12]-[14]. As opposed to the AFI, here the cancerous areas show a higher fluorescent signal with respect to the surrounding healthy tissues[12], [15]. Implementation of fluorescence imaging in capsule endoscopy could potentially exploit both methodologies leading to a more accurate diagnosis of cancer in the GI tract.

A miniaturised system for detecting variation in the concentration of fluorescence dye in aqueous solution was implemented in 2011[16]. Two years later, a tethered fluorescence sensor for detection of fluorescence dyes in the blood stream was developed[17]. A prototype for measuring infrared fluorescence emitted by indocyanine green (IG) used as contrast for cancer detection was demonstrated by Demosthenous et al.[18]. All these systems were developed to offer fluorescence measurements from within the body without offering imaging capabilities. A proof of concept capsule for AFI of the GI tract has been demonstrated [19]. However, it exceeded the acceptable target for capsule endoscopy[20].

${ }^{4}$ Department of Pathology, Alexander Donald Building, Western General Hospital, Crewe Road, University of Edinburgh, EH4 2XU, United Kingdom. 
a)

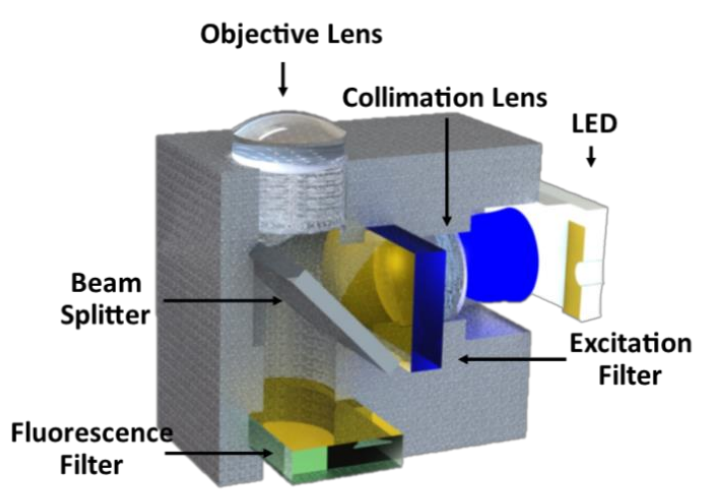

c)

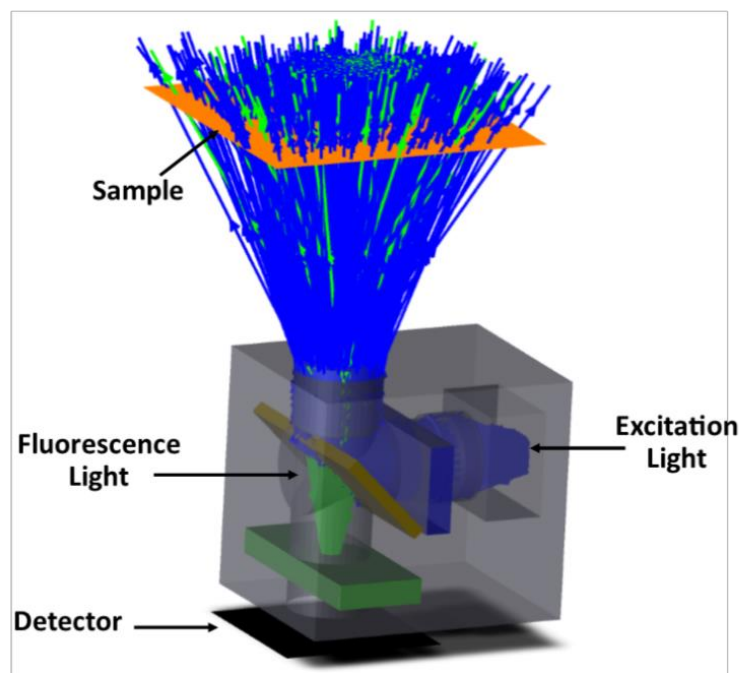

b)

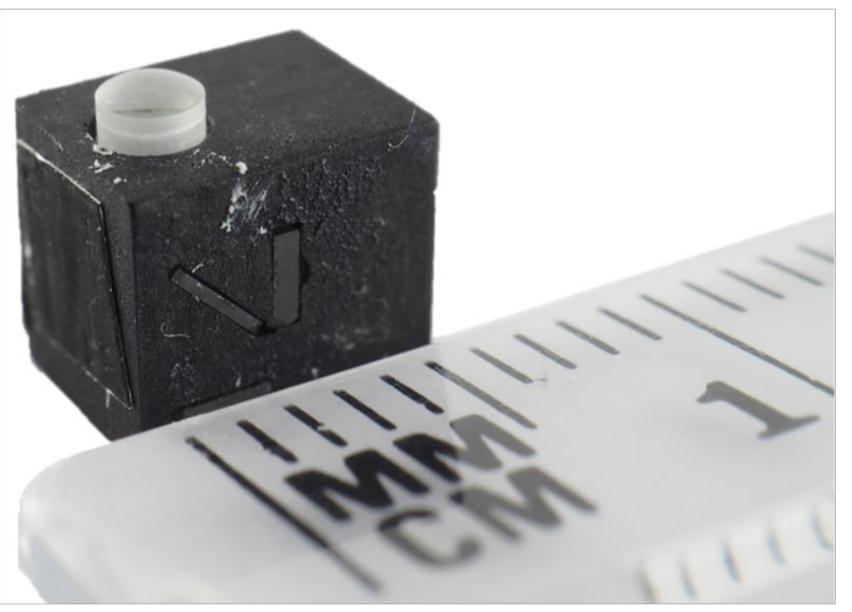

d)

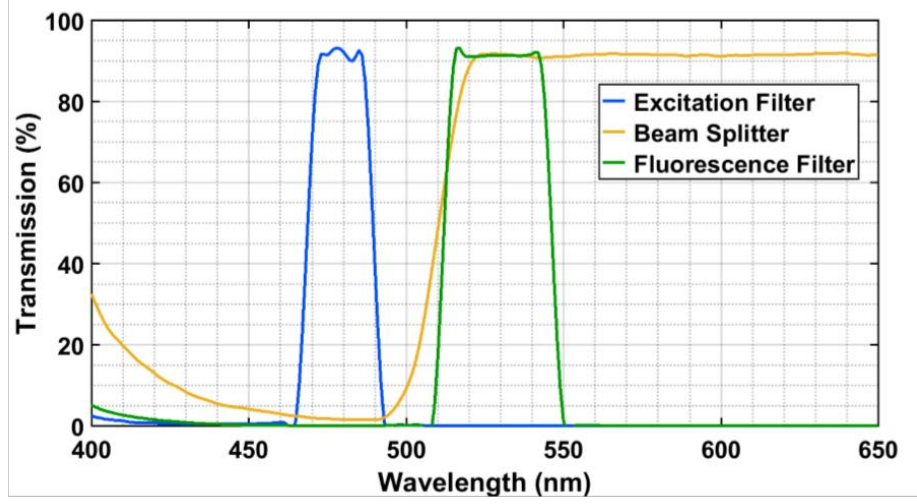

Fig. 1. Miniaturised optical block for fluorescence imaging in capsule endoscopy. (a) Cross-section model of the optical block consisting of an LED, collimation lens, excitation filter, beam splitter, fluorescence filter and objective lens. (b) Final assembled optical block prototype. (c) Optical simulation of the block showing the excitation light from the LED filtered and reflected on the sample and fluorescence light from the sample imaged by the objective lens onto the detector after passing through the fluorescence filter. (d) Optical transmission properties of the excitation filter, beam splitter and fluorescence filter incorporated in the block.

The challenge is to design an optical system capable of imaging small changes in fluorescence signal while being small enough to fit in a capsule that will meet the standard medical requirements[21].

Here we present a low-cost miniaturised optical block ( $5 \mathrm{~mm}$ W x $6 \mathrm{~mm} \mathrm{~L}$ × $5 \mathrm{~mm} \mathrm{H}$ ), capable of performing both AFI and fluorescence labelling imaging, with dimensions suitable for integration in a capsule for endoscopy. The optical unit consists of a LED, miniature lenses and ultrathin optical filters to minimize the interference between excitation and fluorescence light. The imager was completed and tested using a sensitive 64 x 64 Single Photon Avalanche Diode (SPAD) CMOS chip[22]. The system was characterized by imaging phantom FAD solutions. Moreover, fluorescence from colorectal cancer cells labelled with cancer-selective ProteoGREENTM-gGlu was successfully detected. The labelling performance of the cancer selective probe was compared to the performance of the generic fluorescence dye CellTracker ${ }^{\mathrm{TM}}$ Green for monitoring cells movement. Results were validated by using a Modulus ${ }^{\mathrm{TM}}$ microplate reader. Finally, the system was used to image autofluorescence signals from healthy and malignant ex vivo human tissues from the colon.

\section{MATERIALS AND METHODS}

\section{A. Optical Block}

The optical design of the block was realized using Zemax Optics Studio. The body accommodating the optical components was designed using SolidWorks.

The block is made of aluminium and consists of an aperture and slots for fitting lenses and filters. The precise position of the slots was achieved using a standard wire erosion process. The slots were designed with a tolerance of $100 \mu \mathrm{m}$ with respect to the final sizes of the optical components. The block was anodized and painted black to minimize unwanted reflections of light near the optical path (Fig. S1 (a), (b), Supplementary Information).

The final dimensions of the block are $5 \mathrm{~mm} \mathrm{~W} \times 6 \mathrm{~mm} \mathrm{~L} \mathrm{x} 5$ $\mathrm{mm}$ H. The components used were a LED (Broadcom Limited ASMT-BB20-NS000), collimation lens (Edmund Optics), excitation filter (480/17 nm BrightLine $\AA$, Semrock), dichroic beam splitter (506 nm edge BrightLine ${ }^{\circ}$, Semrock), objective lens (84-124, Edmund Optics) and fluorescence filter (529/28 $\mathrm{nm}$ BrightLine $\AA$, Semrock) (Fig. S1 (c), Supplementary Information). The thickness of the as-bought filters was not 
suitable for integration into the block, therefore lapping and polishing were needed to reach the required thickness to optimize miniaturisation (Fig. S2, Supplementary Information). Specifically, the thickness of the excitation and fluorescence filters was reduced from $2 \mathrm{~mm}$ to $500 \mu \mathrm{m}$ whereas the beam splitter thickness was reduced from $1.2 \mathrm{~mm}$ to $200 \mu \mathrm{m}$. Transmission and reflection properties of the filters were measured before and after lapping and polishing by using a micro-spectrophotometer (Foster + Freeman ffTA/MS) to ensure that the fabrication process did not affect their optical performance (Fig. S3 Supplementary Information).

\section{B. $64 \times 64$ CMOS-chip SPAD array}

The SPAD chip was fabricated by AMS AG in a legacy unmodified $180 \mathrm{~nm}$ high voltage (HV) CMOS process. The sensor chip has a $64 \times 64$ array of pixels and was designed using Cadence Virtuoso software package. Each pixel is a SPAD biased in Geiger mode to detect single photons of light impinging the active area. An ST nucleo F338R8 Mbed board was programmed to interface the chip to a laptop and read count events from the chip (Fig. S4, Supplementary Information). A Matlab algorithm synchronized and read the data on a serial port and then reconstructed the data into an image with a frame rate of $1.3 \mathrm{fps}$. The imager array was characterized in previous work[22].

\section{Imaging Setup}

The SPAD chip was mounted on to the side of the optical block using a black nylon disk with an aperture in the centre as a spacer. The module was in turn mounted on to a PCB that also carried the microcontroller and read-out electronics. The imager assembly was mounted on to an optical breadboard. Also located on the breadboard was a set of motorized X-Y-Z optical stages so as to position and manipulate objects to be imaged with respect to the fluorescence imaging cube (Fig. S5, Supplementary Information). The exit pupil of the optical block was $2 \mathrm{~mm}$ in diameter whereas the SPAD imaging array was $3.9 \mathrm{~mm} \times 3.9 \mathrm{~mm}$. Since the outer regions of the array were not used, a software mask was used to exclude the dark outer pixels (Fig. S6, Supplementary Information).

Before imaging any sample, the optical system was placed in an enclosed dark space to evaluate the effect of the noise sources. The Dark Count Rate (DCR) is the number of events a SPAD reads in the absence of light arising from thermal excitation and band-to-band tunnelling. In our imaging system, another component of noise was from stray light as a consequence of the small amount of excitation light coming from the LED that is scattered on to the SPAD. DCR was measured by taking 10 frames in a dark environment with the LED turned off. The same process was repeated with the LED turned on to evaluate the noise introduced by the LED into the system. The ten frames were averaged in a single frame. All experiments were performed at fourteen different sample intervals for the SPADs in the array[22]. The average noise was computed only for the pixels within the aforementioned software mask. The DCR and stray-light noise plot is shown in figure S7, in the supplementary information section.

\section{Fluorescence Phantom Solutions Experiments}

Flavin adenine dinucleotide salt hydrate (molecular mass $829.5 \mathrm{~g} / \mathrm{mol}$, purity $>95 \%$ Sigma Aldrich) was dissolved in Phosphate Buffer Saline (PBS) to obtain six separate phantom solutions at concentrations of $250 \mu \mathrm{M}, 125 \mu \mathrm{M}, 60 \mu \mathrm{M}, 30 \mu \mathrm{M}$, $15 \mu \mathrm{M}$, and $7.5 \mu \mathrm{M}$ respectively.

A 3" x 3" Positive Fluorescent, 1951 USAF Target mask (Edmund Optics) was placed on the objective stage. The mask consisted of a layer of chromium into which transparent features were etched.

$10 \mu \mathrm{L}$ Flavin solutions were separately pipetted on to a 625 $\mu \mathrm{m}$ x $625 \mu \mathrm{m}$ open square window on the USAF mask. The six separate solutions with different concentrations were imaged using the imager. The average of all the pixels within the fluorescence feature was computed for each concentration. Experiments were replicated three times per concentration. The software mask described previously in the text was subtracted from the images in order to account only for the fluorescence signal emitted by the phantom solutions.

\section{E. In vitro Experiments}

CellTracker ${ }^{\mathrm{TM}}$ Green CMFDA Dye is a fluorescence dye for monitoring cell location designed to work with all cell types. It was purchased from Thermofisher and dissolved in $20 \mu \mathrm{L}$ of anhydrous dimethyl sulfoxide (DMSO) to make a stock solution of $1 \mathrm{mM}$. The stock solution was diluted in Hank balanced salt solution (HBSS) to make $1 \mu \mathrm{M}, 2 \mu \mathrm{M}$ and $5 \mu \mathrm{M}$ cell stain solution.

Cancer-selective probe ProteoGreen ${ }^{\mathrm{TM}_{-} \mathrm{gGlu} \text { was purchased }}$ from Goryo Chemical and dissolved in $29.7 \mu \mathrm{L}$ of DMSO to prepare a $1 \mathrm{mM}$ stock solution. The stock solution was diluted with HBBS to make $1 \mu \mathrm{M}, 2 \mu \mathrm{M}$ and $5 \mu \mathrm{M}$ cell stain solution.

Dukes' type B colorectal adenocarcinoma cell line LS 174T (ATCC ${ }^{\circledR}$ CL-188 ${ }^{\mathrm{TM}}$ ) and human hTERT-immortalized foreskin fibroblast cell line BJ-5ta (ATCC ${ }^{\circledR}$ CRL-4001 ${ }^{\mathrm{TM}}$ ) where purchased and sub-cultured in two separate $75 \mathrm{~cm}^{2}$ flasks at $37^{\circ} \mathrm{C}$ in $5 \% \mathrm{CO} 2$ atmosphere. The fibroblasts were used as negative control for the cancer-selective probe.

Cultures with specified seeding densities of 100000 cells $/ \mathrm{cm}^{2}, 25000$ cells $/ \mathrm{cm}^{2}, 6250$ cells $/ \mathrm{cm}^{2}$, and 1562 cells $/ \mathrm{cm}^{2}$ were replicated four times each within a 96 well plate (Fig. S8 (a), Supplementary Information). Four additional wells per plate were pipetted with culture medium only, for control purposes. The protocol was followed using separate plates for hTERT-immortalized foreskin fibroblast and Dukes' type B colorectal adenocarcinoma cell populations. The protocol is pictured in figure S8 (a) of the supplementary information section. ProteoGreenTM-gGlu and CellTracker ${ }^{\mathrm{TM}}$ Green were used to label plates cultured with the adenocarcinoma cancer cells prepared following the aforementioned culture protocol. Three separate plates for each dye were labelled with concentrations of $5 \mu \mathrm{M}, 2 \mu \mathrm{M}$ and $1 \mu \mathrm{M}$ respectively. Three other plates were not stained to assess the fluoresce signal from cells without any fluorescence label. Moreover, three plates cultured with the negative control hTERT-immortalized foreskin fibroblast cells were also separately labelled with concentrations of $5 \mu \mathrm{M}, 2 \mu \mathrm{M}$ and $1 \mu \mathrm{M}$ of ProteoGreen ${ }^{\mathrm{TM}}-\mathrm{gGlu}$. 
a)
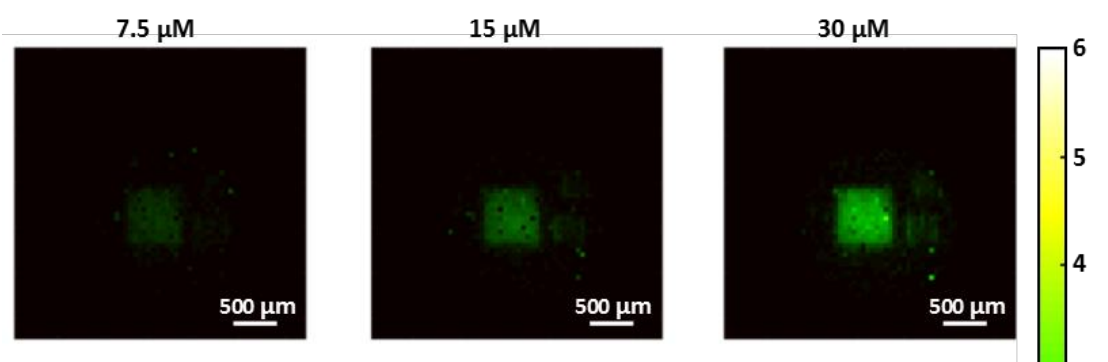

$60 \mu \mathrm{M}$
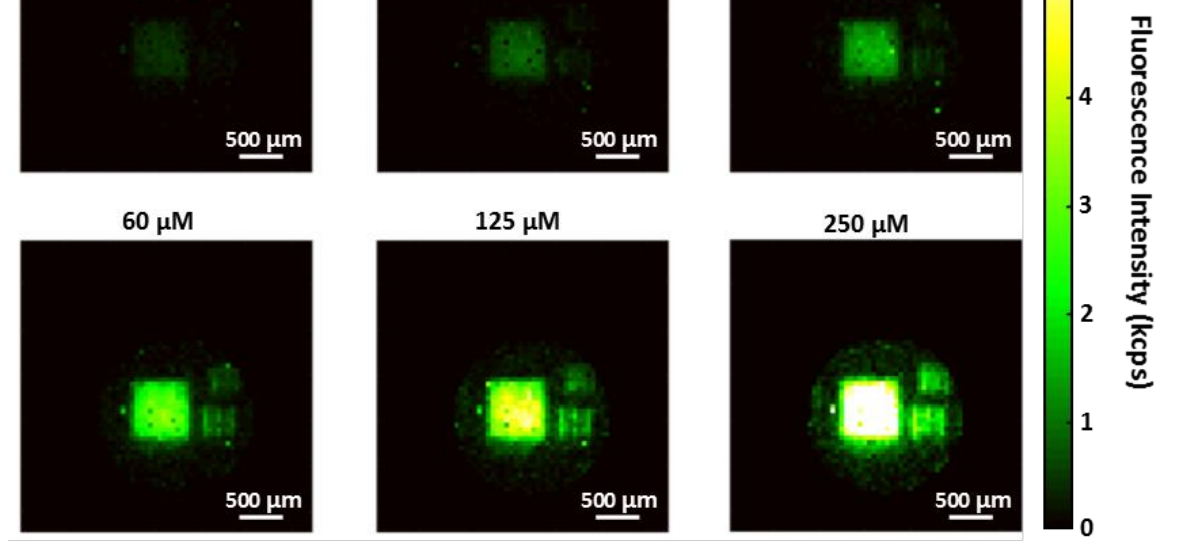

b)

c)
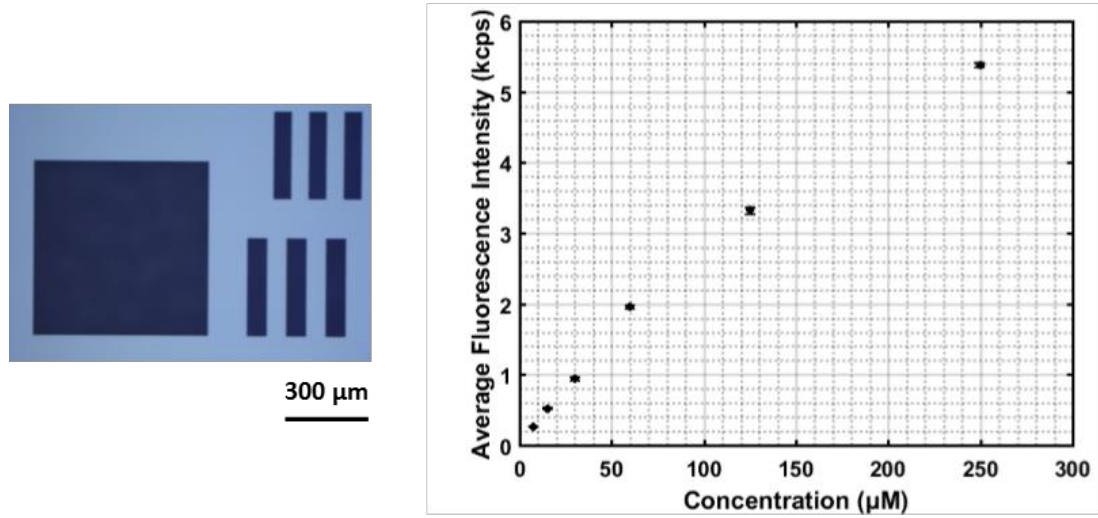

Fig. 2 Response of the optical system to FAD at six different concentrations. (a) Images of FAD solutions masked by the feature of the USAF target at concentrations of $7.5 \mu \mathrm{M}, 15 \mu \mathrm{M}, 30 \mu \mathrm{M}, 60 \mu \mathrm{M}, 125 \mu \mathrm{M}, 250 \mu \mathrm{M}$. (b) Imaging feature in the USAF target (c) Average fluorescence intensity of the pixels within the square feature for each concentration was computed to assess the optical sensitivity of the system as a function of concentration. The data shows the mean of independent experiments $(n=3)$ and the error bars show the standard deviation.

The optical fluorescence signal from the cells stained with the fluorescence probes was expected to change as a function of the cell density in the wells. Since the field of view of the imager was $2 \mathrm{~mm}$ and the well diameter was $6.3 \mathrm{~mm}$, it was necessary to scan the wells over the imager to capture a representative signal (Fig. S8 (b), Supplementary Information). The data from each pixel in each of the frame that made a single scan were averaged together. This procedure was also carried out for the wells containing only culture medium to determine control replicate $\overline{\boldsymbol{M}}$ which was subtracted from the data. For each plate, the average fluorescence value $\overline{\boldsymbol{\mu}}_{(s)}$ where s denotes the seeding density was calculated by averaging the values from all four wells cultured with the same seeding densities within the same plate. Three plates for each concentration of dye were measured. The final fluorescence value for each seeding density at a specific dye concentration was computed by averaging the values obtained from the three experiments at the specific seeding density. The procedure was also performed on three plates without any labelling probes to assess the fluorescence from the unstained cells at the aforementioned four seeding densities. The same experimental procedure was performed by using the Modulus ${ }^{\mathrm{TM}}$ Microplate reader. In this case, no scanning was required as the system directly provided a representative fluorescence signal from each well.

\section{F. Ex-vivo Experiments}

Appropriate institutional ethical consent and pre-operative patient consent were obtained for the acquisition of tissue specimens. Portions of normal colon and cancer tissue $\left(5 \mathrm{~mm}^{2}\right)$ were collected fresh, within 30 minutes, from patients undergoing surgical resection (right hemicolectomy) for adenocarcinoma of the colon. Before imaging any tissue, images of the surface of an empty petri dish were acquired to account for any reflection from the petri dish. The average of the frames of the petri dish surface was saved to use as a mask to subtract to the tissues images. Healthy and cancer tissues from the same colon sample were placed in the same petri dish in succession. Optical images of the tissues were acquired by using a portable optical microscope (Dino-Lite AM3113T USB 
Digital Microscope). Finally, a square area of the petri dish surrounding both the tissues was raster scanned on top of the optical system. Images were acquired for the full duration of the scan. The complete image of the tissues was reconstructed by horizontal and vertical superimposition of the frames acquired during the scan (Fig. S9, Supplementary Information).

\section{RESULTS AND DISCUSSION}

\section{A. Optical System for Fluorescence Imaging}

A miniaturised optical block for fluorescence imaging in capsule endoscopy is presented. The block is made of aluminium and was realized by wire erosion process. It accommodates an LED, two lenses and three optical filters to separate excitation and green fluorescence light (Figs. 1a, 1b). The optics were designed to successfully separate the excitation light from green fluorescence light between 520 and $530 \mathrm{~nm}$. As it is shown in Fig. 1 (c), in excitation mode, light from the LED, with a center wavelength of $468 \mathrm{~nm}$ and a $15^{\circ}$ beam angle, was collimated and filtered onto the beam splitter placed at a $45^{\circ}$ angle. The excitation beam was reflected through the objective lens to the sample. In fluorescent mode, the fluorescent emission from the sample was imaged by the achromatic objective lens after passing through the fluorescence emission filter. The imager was completed using a $64 \times 64$ pixel SPAD array. The miniaturized imager could be easily integrated in capsules for endoscopy to image and detect small variations in the autofluorescence signal of the GI walls. Such measurements can be crucial for early cancer diagnosis. The power consumption of each component makes the system suitable for the integration in capsule format by using charge pump technology [23], [24]. The LED and the SPAD array consume $3 \mathrm{~mA}$ and $5 \mathrm{~mA}$ respectively. With a $3 \mathrm{~V}, 150 \mathrm{mAh}$ battery the system will operate for approximately 17 hours. The full imaging system described in the material and methods section was characterised by evaluating the crosstalk noise component which is the small amount of excitation light coming from the LED scattering on to the active area of the SPAD (Supplementary Information S7). The average crosstalk noise from the LED was $435 \mathrm{cps}$.

\section{B. Imaging of FAD Phantom Solution}

As discussed in both the introduction and materials and methods sections, FAD is an endogenous fluorophore expressed in human tissues. Measurements were carried out on FAD salt hydrate dissolved in phosphate buffer saline to determine the minimum concentration of fluorophore in solution detectable by the system. Variation in the ratio between the oxidized form of $\mathrm{FAD}$, with fluorescence emission peak between 525 and $530 \mathrm{~nm}$, and its reduced form $\left(\mathrm{FADH}_{2}\right)$, which does not emit any fluorescence, are directly linked to the metabolic activity within the tissues during carcinogenesis[25]. The increase of $\mathrm{FADH}_{2}$ over FAD is one of the causes of a reduced autofluorescence signal from cancer tissue [26]. Using a $625 \mu \mathrm{m} \times 625 \mu \mathrm{m}$ feature in an USAF target to form a regular mask, the imaging system was tested to measure the fluorescence signal as a function of concentration. As can be seen in Fig. 2, count rates from the pixels within the square feature were reliably calculated indicating that the smallest measurable concentration was as little as $7.5 \mu \mathrm{M}$.

\section{Detection of fluorescence-labelled colorectal cancer cells}

Accuracy in tumour diagnosis and assessment of cancer lesions is critical to disease treatment. A precise early detection of cancer can be achieved by targeting specific cells or molecules that are overexpressed and metabolized in tissues undergoing carcinogenesis[27]. In this work, we used a Gamma-glutamyl transpeptidase (GGT) selective fluorescence probe $\gamma$-glutamyl hydroxymethyl rhodamine green (gGluHMRG) that is sold as ProteoGreenGlu ${ }^{\mathrm{TM}}$ [16]. GGT is an enzyme expressed on cells membranes acting as catalyst in the cellular glutathione homeostasis. GGT expression is high on the membranes of several cancer cells and low in normal tissues. gGluHMRG does not show any fluorescence under low levels of GGT. However, the reaction with GGT on the membranes of cancer cells leads to the hydrolysis of gGluHMRG into hydrophobic HMRG which has a fluorescence signal with a peak at $525 \mathrm{~nm}$. HMRG can freely pass through cells membranes and accumulate in lysosomes making the cells fluorescent. We assessed the capability of our optical system to detect fluorescence from colorectal cancer cells cultured in a 96 well plate at four different seeding densities and separately labelled with Proteogreen $\mathrm{gGlu}^{\mathrm{TM}}$ at three different concentrations. Fluorescence intensities from four wells cultured with the same cell line and labelled with the fluorescence dye Cell Tracker Green ${ }^{\mathrm{TM}}$, which is not cancer specific, were also measured to further characterise the system. A Modulus ${ }^{\mathrm{TM}}$ Microplate was used as reference instrument to measure fluorescence intensities from the cells and validate our device. Figure $3(\mathrm{a}, \mathrm{c})$ shows the response of the imaging system and the Modulus ${ }^{\mathrm{TM}}$ instrument demonstrating an increase in the fluorescence signals from all the four seeding densities after being labelled with CellTrackerGreen ${ }^{\mathrm{TM}}$. The cancer-selective probe Proteogreen $\mathrm{gGlu}^{\mathrm{TM}}$ demonstrated a lower increase of the fluorescence signal than the CellTrackerGreenTM. As we can see from figure $3(\mathrm{~b}, \mathrm{~d})$, a clear fluorescence signal is obtained for seeding densities greater than $2 \times 104 \mathrm{cell} / \mathrm{cm}^{2}$. The specificity of Proteogreen $\mathrm{gGlu}^{\mathrm{TM}}$ for cancer cells was also validated by labelling a non-cancerous cell line of fibroblasts immortalized with hTERT. Figure 4 shows that there is no obvious increase in fibroblasts, labelled with $5 \mu \mathrm{M}$ of the probe. We also directly compared the data from the miniaturised system and the Modulus ${ }^{\mathrm{TM}}$ microplate. As can be seen from figures S10 and S11 in the supplementary information section, the result is a linear plot with a correlation R $2>90 \%$ at each concentration of the dyes indicating that our device has equivalent response to the large-scale commercialised system. 
a)

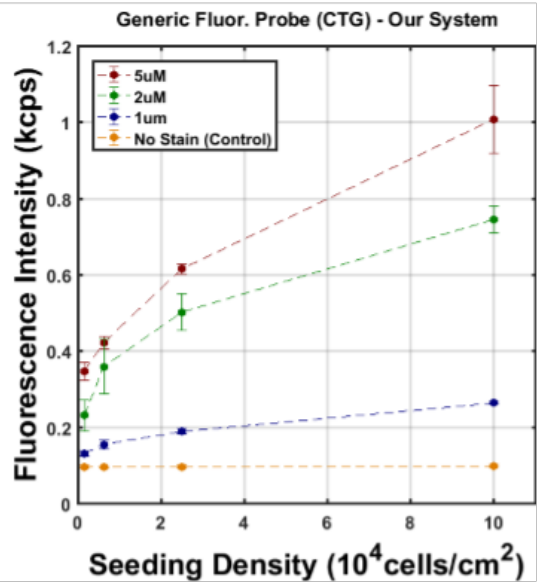

c)

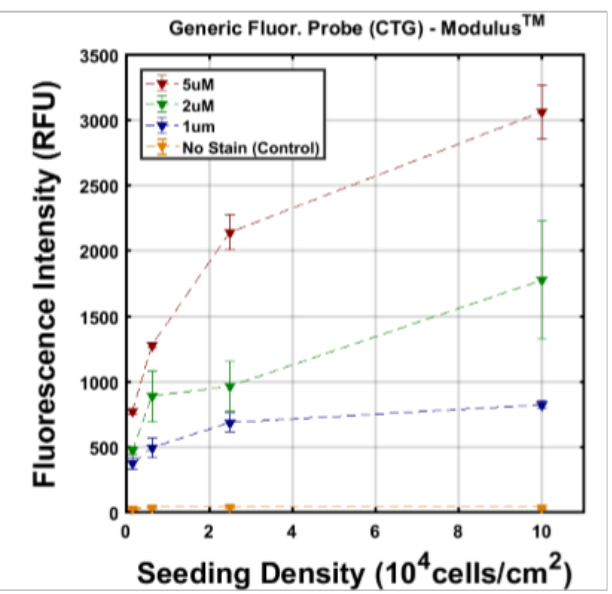

b)

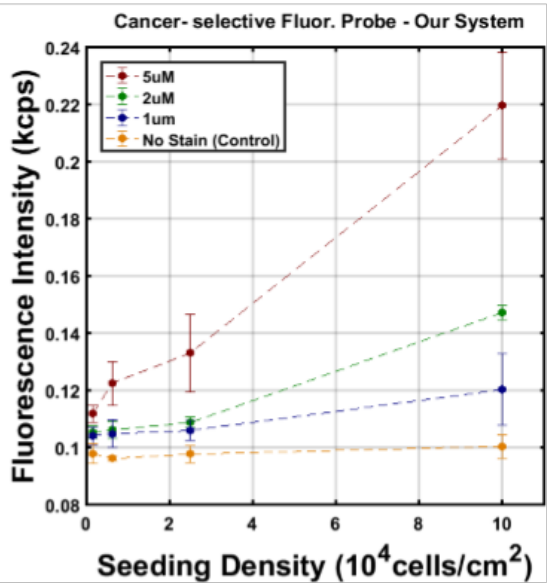

d)

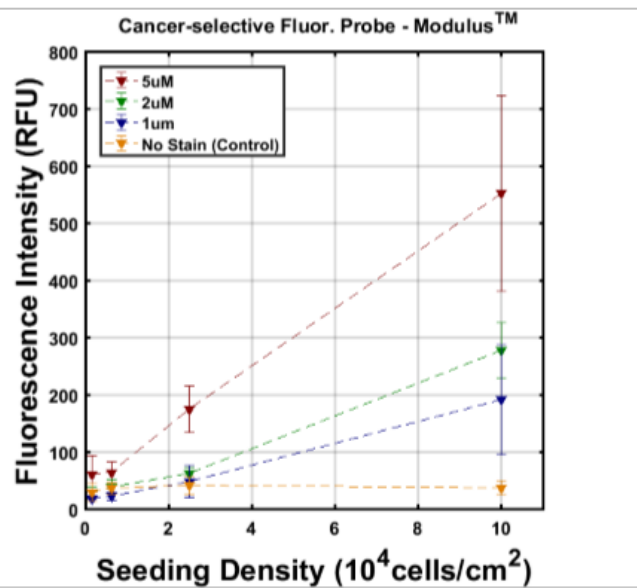

Fig. 3: Comparison of optical block and SPAD with Modulus ${ }^{\mathrm{TM}}$ microplate for detection of fluorescence from colorectal cancer cells cultured at seeding densities of 1562 cells $/ \mathrm{cm}^{2}, 6250$ cells $/ \mathrm{cm}^{2}, 25,000$ cells $/ \mathrm{cm}^{2}, 100,000 \mathrm{cells} / \mathrm{cm}^{2}$ and separately labelled with generic fluorescence probe (CellTrackerTM Green CMFDA) and cancer-selective fluorescence probe (Proteogreen gGluTM). (a) Detection of fluorescence in colorectal cancer cells labelled with fluorescence probe CellTracker ${ }^{\mathrm{TM}}$ Green CMFDA at concentration of $5 \mu \mathrm{M}, 2 \mu \mathrm{M}$ and $1 \mu \mathrm{M}$ using our system. (b) Detection of fluorescence in colorectal cancer cells labelled with cancer selective fluorescence probe Proteogreen $\mathrm{gGlu}^{\mathrm{TM}}$ at concentration of $5 \mu \mathrm{M}, 2 \mu \mathrm{M}$ and $1 \mu \mathrm{M}$ using our system. (c) Detection of fluorescence in colorectal cancer cells labelled with fluorescence probe CellTracker ${ }^{\mathrm{TM}}$ Green CMFDA at concentration of $5 \mu \mathrm{M}, 2 \mu \mathrm{M}$ and $1 \mu \mathrm{M}$ using Modulus ${ }^{\mathrm{TM}}$ microplate (d) Detection of fluorescence in colorectal cancer cells labelled with fluorescence probe Proteogreen $\mathrm{gGlu}^{\mathrm{TM}}$ at concentration of $5 \mu \mathrm{M}, 2 \mu \mathrm{M}$ and $1 \mu \mathrm{M}$ using Modulus ${ }^{\mathrm{TM}}$ microplate. Each seeding density was cultured four times in a 96 wells plate. All four wells were labelled and fluorescence value from each well was measured. The average of the four values from a plate was considered as an experimental replicate. The experiments were replicated in three different plates for a total of three replicates per concentration of probe. Fluorescence intensities from three plates of cells without any fluorescence labelling were also measured.

a)

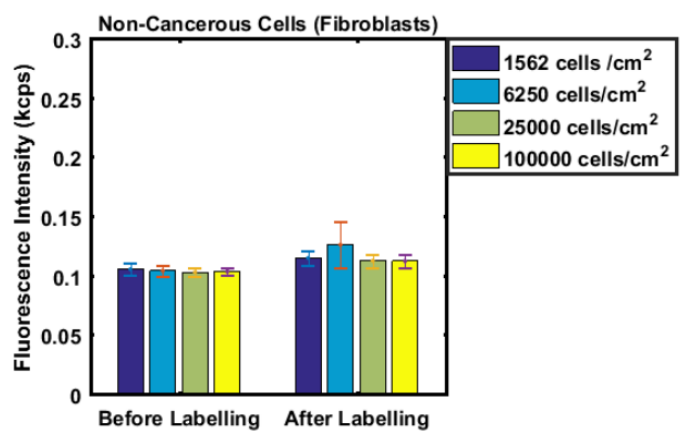

b)

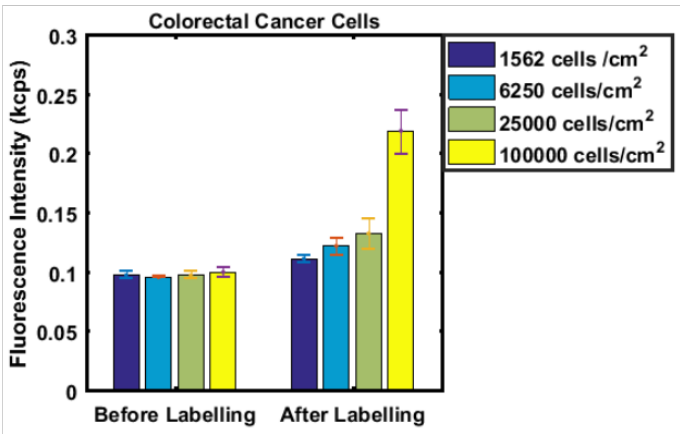

Fig. 4: Response of optical system for capsule endoscopy to fibroblasts labelled with $5 \mu \mathrm{M}$ of the cancer-selective fluorescence probe (Proteogreen gGlu ${ }^{\mathrm{TM}}$ ) in comparison to colorectal cancer cells labelled with the same probe. (a) The plot depicts the measured values of fluorescence intensity from fibroblasts at seeding densities of 1562 cells $/ \mathrm{cm}^{2}, 6250$ cells $/ \mathrm{cm}^{2}, 25,000$ cells $/ \mathrm{cm}^{2}$ and 100,000 cells $/ \mathrm{cm}^{2}$ before and after fluorescence labelling with Proteogreen gGluTM. (b) The plot depicts the measured values of fluorescence intensity from colorectal cancer cells at seeding densities of 1562 cells $/ \mathrm{cm}^{2}, 6250 \mathrm{cells} / \mathrm{cm}^{2}, 25,000 \mathrm{cells} / \mathrm{cm}^{2}$ and $100,000 \mathrm{cells} / \mathrm{cm}^{2}$ after fluorescence labelling with $5 \mu \mathrm{M}$ of Proteogreen $\mathrm{gGlu}{ }^{\mathrm{TM}}$. The increase in the fluorescence signal from colon cancer cell line after labelling is more pronounced at the highest seeding density. The experiments were replicated in three different plates. 
a) Patient 1

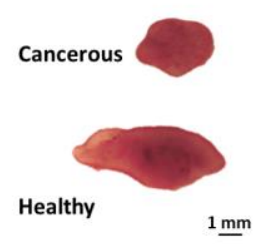

Patient 1 : AF Image

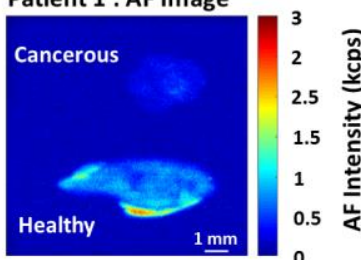

b) Patient 2

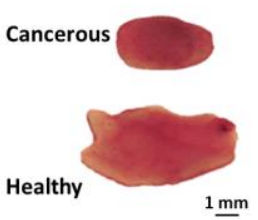

Patient 2 : AF Image

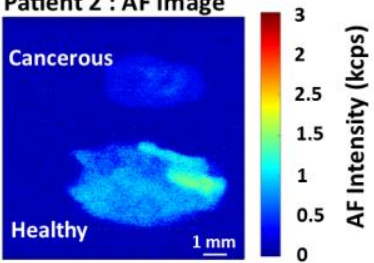

c) Patient 3

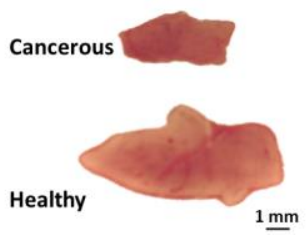

d) Patient 4

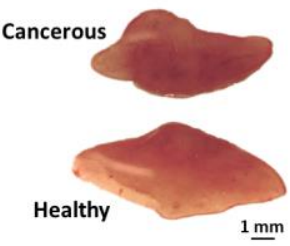

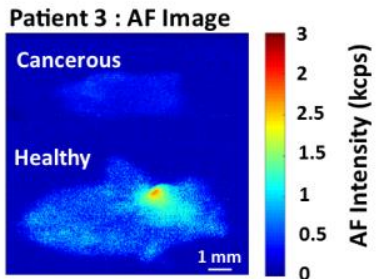

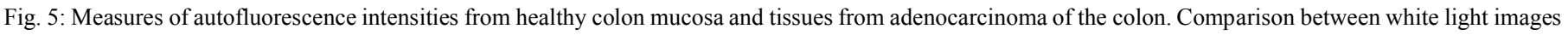
and autofluorescence (AF) images of human adenocarcinoma tissue and human healthy colon mucosa from four different patients (a), (b), (c), (d). AF images were

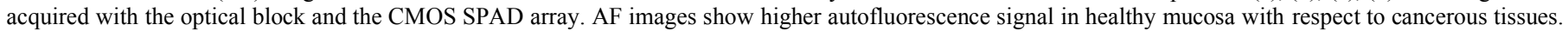

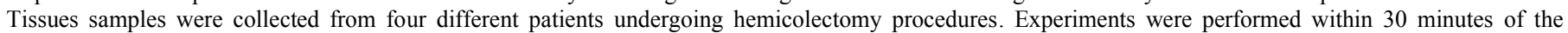

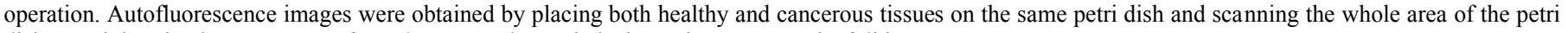
dish containing the tissues. Frames from the scans where stitched together to create the full images.

\section{Autofluorescence imaging of human healthy colon mucosa and human adenocarcinoma of the colon}

Samples from four different patients with adenocarcinoma of the colon were collected after hemicolectomy to obtain autofluorescence images of healthy colon mucosa and tumour tissues. Healthy colon mucosa has three main layers defined as mucosa, submucosa and muscolaris propria. Submucosa is reported to be the most fluorescent component[28].

Autofluorescene images from eight samples revealed differences in the clinically measured autofluorescence intensities between normal and tumour tissue (Fig. 5). No difference could be seen in the white light optical images. The autofluorescence from healthy colon mucosa was always higher.

We attribute the observed data to increased haemoglobin absorption in cancerous tissues as well as to the fact that carcinogenesis is responsible for an increase in thickness of the tumour mucosa and thus a decrease in the fluorescence signal from the submucosa[29]. It has been shown that gGlu-HMRG is not effective when used to label resected colon cancer tissues hence we did not study its performance in this work[12].

\section{CONCLUSION}

Fluorescence imaging has the potential to significantly improve capsule endoscopy by reducing the error rate in the detection of cancer. We have developed a fluorescence imager with dimensions suitable for integration into capsule endoscopy. The optical module was fabricated by using widely available and low-cost manufacturing processes. The system comprising a new miniaturised optical block and a 64 x 64 SPAD array was used to detect green fluorescence from FAD that is an endogenous fluorophore responsible for autofluorescence in human tissues. Fluorescence from colorectal cancer cells labelled with a cancer specific molecular probe was detected at three different concentrations of the labelling-probe. The results we obtained compared favourably with a large bench-top conventional system. Clinical studies performed using resected malignant tissues from the human colon demonstrated a clear distinction in endogenous autofluorescence between normal and malignant tissues. We attribute the reduction in the signal to either conversion of FAD to $\mathrm{FADH}_{2}$ in the tumour or to the thickening of the mucosal layer either of which is associated with cancer tissues. Increased presence of haemoglobin as a consequence of vascularization in tumours may also have been a factor. The highly miniaturised nature of the device demonstrates the technology's potential use in capsule endoscopy

\section{ACKNOWLEDGMENT}

This work was funded by the centre for doctoral training (CDT) in intelligent sensing and measurements (ISM) and was carried out as part of the SONOPILL project funded by the Engineering and Physical Sciences Research Council (EPSRC), UK (EP/K034537/1).

\section{REFERENCES}

[1] Gavriel Iddan, Gavriel Meron, Arkady Glukhovsky, and Paul Swain, "Wireless capsule endoscopy," Nature, vol. 405, no. 6785, 2000.

[2] B. S. Lewis, G. M. Eisen, and S. Friedman, “A pooled analysis to evaluate results of capsule endoscopy trials," Endoscopy, vol. 37, no. 10, p. 960-965, Oct. 2005.

[3] J. Haringsma et al., "Autofluorescence endoscopy: Feasibility of detection of GI neoplasms unapparent to white light endoscopy with an evolving technology," Gastrointest. Endosc., vol. 53, no. 6, pp. 642-650, 2001. 
[4] W. L. Curvers et al., "Endoscopic tri-modal imaging for detection of early neoplasia in Barrett's oesophagus : a multi-centre feasibility study using high-resolution endoscopy , autofluorescence imaging and narrow band imaging incorporated in one endoscopy system."

[5] "World's First Gastrointestinal Videoendoscopes with Auto Fluorescence Imaging Capability."

[6] S. M. Bae et al., "Multi-Spectral Fluorescence Imaging of Colon Dysplasia InVivo Using a MultiSpectral Endoscopy System," Transl. Oncol., vol. 12, no. 2, pp. 226-235, Nov. 2018.

[7] K. Moriichi, M. Fujiya, and T. Okumura, "The efficacy of autofluorescence imaging in the diagnosis of colorectal diseases," Clin. J. Gastroenterol., vol. 9, no. 4, pp. 175-183, 2016.

[8] M. Filip, S. Iordache, A. Săftoiu, and T. Ciurea, "Autofluorescence imaging and magnification endoscopy.," World J. Gastroenterol., vol. 17, no. 1, pp. 9-14, Jan. 2011.

[9] Y. Urano et al., "Rapid Cancer Detection by Topically Spraying a g -Glutamyltranspeptidase - Activated Fluorescent Probe," vol. 3, no. 110, 2011.

[10] Q. T. Nguyen, E. S. Olson, T. A. Aguilera, T. Jiang, M. Scadeng, and L. G. Ellies, "Surgery with molecular fl uorescence imaging using activatable cellpenetrating peptides decreases residual cancer and improves survival," pp. 1-6, 2009.

[11] T. Terai and T. Nagano, "Small-molecule fluorophores and fluorescent probes for bioimaging," pp. 347-359, 2013.

[12] H. Matsuzaki, M. Kamiya, R. J. Iwatate, D. Asanuma, T. Watanabe, and Y. Urano, "Novel HexosaminidaseTargeting Fluorescence Probe for Visualizing Human Colorectal Cancer," Bioconjug. Chem., vol. 27, no. 4, pp. 973-981, Apr. 2016.

[13] M. Mitsunaga et al., "Fluorescence endoscopic detection of murine colitis-associated colon cancer by topically applied enzymatically rapid-activatable probe," pp. 1179-1186, 2013.

[14] K. Kawakami et al., "Gamma-glutamyltransferase activity in exosomes as a potential marker for prostate cancer," pp. 1-12, 2017.

[15] C. Sato et al., "A Pilot Study of Fluorescent Imaging of Colorectal Tumors Using a \&\#947;-GlutamylTranspeptidase-Activatable Fluorescent Probe," Digestion, vol. 91, no. 1, pp. 70-76, 2015.

[16] M. Ryou et al., "An implantable wireless biosensor for the immediate detection of upper GI bleeding : a new fluorescein-based tool for diagnosis and surveillance ( with video )," YMGE, vol. 74, no. 1, pp. 189-194.e1, 2011.

[17] T. D. O. Sullivan et al., "Real-time, continuous, fluorescence sensing in a freely-moving subject with an implanted hybrid VCSEL / CMOS biosensor," vol. 4, no. 8, pp. 1332-1341, 2013.

[18] P. Demosthenous, C. Pitris, J. Georgiou, and S. Member, "Infrared Fluorescence-Based Cancer Screening Capsule for the Small Intestine," IEEE Trans. Biomed. Circuits Syst., vol. 10, no. 2, pp. 467-
476, 2016.

[19] M. a. Al-Rawhani, J. Beeley, and D. R. S. Cumming, "Wireless fluorescence capsule for endoscopy using single photon-based detection," Sci. Rep., vol. 5, no. November, p. 18591, 2015.

[20] Z. Li, Z. Liao, and M. McAlindon, Handbook of capsule endoscopy. 2014.

[21] A. Nemeth, Role of capsule endoscopy in small bowel management. Lund University, 2019.

[22] C. Accarino et al., "A 64x64 SPAD array for portable colorimetric sensing, fluorescence and x-ray imaging," IEEE Sens. J., p. 1, 2019.

[23] L.-R. Dung and Y.-Y. Wu, "A wireless narrowband imaging chip for capsule endoscope," IEEE Trans. Biomed. Circuits Syst., vol. 4, no. 6, pp. 462-468, 2010.

[24] X. Chen et al., "A wireless capsule endoscope system with low-power controlling and processing ASIC," IEEE Trans. Biomed. Circuits Syst., vol. 3, no. 1, pp. $11-22,2009$.

[25] W. Becker, A. Bergmann, R. S. Ibarrola, P.-F. Müller, and L. Braun, "Metabolic imaging by simultaneous FLIM of NAD(P)H and FAD," in Proc.SPIE, 2019, vol. 10882, no.

[26] O. I. Kolenc and K. P. Quinn, "Evaluating Cell Metabolism Through Autofluorescence," vol. 30, no. 6, pp. 875-889, 2019.

[27] H. Kobayashi and P. L. Choyke, "Target-cancer-cellspecific activatable fluorescence imaging probes: rational design and in vivo applications.," Acc. Chem. Res., vol. 44, no. 2, pp. 83-90, Feb. 2011.

[28] G. Bottiroli, A. C. Croce, and D. Locatelli, "Natural Fluorescence of Normal and Neoplastic Human Colon: A Comprehensive 'Ex Vivo' Study,” vol. 60, 1995.

[29] Z. A. Steelman, D. S. Ho, K. K. Chu, and A. Wax, "Light-scattering methods for tissue diagnosis," Optica, vol. 6, no. 4, pp. 479-489, Apr. 2019. 